\title{
Humedales en dolina del norte de Quintana Roo, México: ecosistemas poco conocidos
}

\section{Sinkhole wetlands of the north of Quintana Roo, Mexico: ecosystems barely known}

\author{
Eduardo Cejudo $^{1 *}$ (], K. Geraldine Herrera-Caamal ${ }^{1}$ \\ ${ }^{1}$ Centro de Investigación Científica de Yucatán A.C. Unidad de Ciencias del Agua. Calle 8, No. 39, Mz. 29, S.M. 6., CP. 77500. Teléfono: \\ (998)2113008 ext. 121. Cancún, Quintana Roo, México \\ *Autor de correspondencia: eduardo.cejudo@cicy.mx
}

Artículo científico recibido: 07 de mayo de 2018 aceptado: 18 de enero de 2019

RESUMEN. Los humedales son los ecosistemas más productivos y amenazados del mundo. Si se encuentran en regiones donde domina la roca caliza se denominan humedales cársticos, un tipo de este humedal son los humedales en dolina. EI objetivo del estudio fue caracterizar humedales en dolina del Norte de Quintana Roo, México. Se usaron descriptores de microtopografía, parámetros edáficos, fisicoquímica del agua y biomasa arbórea. Los resultados indican que los humedales en dolina son unidades primarias tipo P/b/h, sistema Palustre, subsistema depresión, clase estacional a intermitentemente inundado, forma ovoide o redonda con vegetación dominada por árboles tolerantes a la inundación en fondo rocoso con sustrato no consolidado. Se encontró mineralización de la materia orgánica, suelo con alta capacidad de retención de agua y elevado contenido de calcio y sílice. La biomasa se estimó entre 0.01 y $0.30 \mathrm{~kg}$ de carbono por árbol.

Palabras clave: Clasificación, fisicoquímica, microtopografía, suelo, volumen maderable.

ABSTRACT. Wetlands are the most productive ecosystems in the world, yet the most endangered. The wetlands established in limestone-dominated areas are referred to as karstic wetlands; a special type of karstic wetlands are sinkhole wetlands. The objective of this study was to characterize sinkhole wetlands of the north of the state of Quintana Roo, Mexico. We used descriptors of the micro-relief, edaphic parameters, water chemistry and tree standing biomass. The results show that sinkhole wetlands are primary units of the type $\mathrm{P} / \mathrm{b} / \mathrm{h}$, swamp depressions from seasonally to intermittently flooded, shape from round to ovoid, dominated by trees tolerant to flooding, established on rocky bottoms with unconsolidated sediments. Surface and interstitial water measurements suggest mineralization of organic matter, soil with high water retention capacity and elevated calcium and silica. Estimated tree standing median biomass was from 0.01 to $0.3 \mathrm{~kg}$ of carbon per tree.

Key words: Classification, physicochemistry, micro-relief, soil, timber volume.

\section{INTRODUCCIÓN}

Los humedales se encuentran entre los ecosistemas más productivos y benéficos del planeta (Cronk y Mitsch 1994, MEA 2005), pero son los más amenazados por la pérdida de superficie y actividades humanas (Ramsar 2015). En estos ecosistemas la biota y los procesos fisicoquímicos del suelo están adaptados a la inundación temporal o permanente, y tienen algunos de los servicios ecosistémicos más importantes (Mitsch y Gosselink 2007). Proporcionan agua en cantidad adecuada y calidad, mantienen biodiversidad, regulan disturbios, almacenan carbono, regulan gases atmosféricos y son sitios de recreación, entre otros (Costanza et al. 2014, Mitsch et al. 2015). En México, se han decretado 142 humedales de importancia internacional, que comprenden 8.6 millones de hectáreas (Ramsar 2018); no obstante, se excluyen humedales urbanos y de poca extensión superficial (Serran y Creed 2016). En zonas de alto desarrollo urbano, el valor por unidad de superficie de algunos humedales aumenta debido al uso de suelo urbano, además son sitios importantes para reducir o amortiguar inundaciones, mejorar la calidad del agua y proveen espacios de recreación (Mitsch y Gosselink 2000).

Los humedales cársticos se encuentran en regiones donde domina roca caliza con proce- 


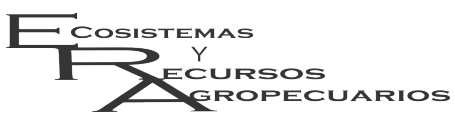

Cejudo y Herrera-Caamal

Humedales en dolina: ecosistemas poco conocidos

Ecosist. Recur. Agropec. 6(17):207-218,2019 sos de disolución que incrementan la porosidad y permeabilidad de la roca con fisuras, fracturas y conductos de disolución (Kresic 2013). En México, los humedales cársticos más conocidos son los petenes (Beltram 2016) y en menor proporción los bajos inundables y sabanas (Palacio Aponte et al. 2002). Los humedales en dolina, conocidos como cenotes, corchales o aguadas, son geoformas generadas por la disolución de roca que causa colapso de la misma, formando oquedades cónicas o de caverna, exponiendo el agua subterránea a la atmósfera (Gutiérrez et al. 2007, Torrescano-Valle y Folan 2015).

La Península de Yucatán cuenta con una gran superficie ocupada por depresiones cársticas donde se pueden establecer humedales en dolina (Aguilar et al. 2016). Los humedales en dolina de regiones templadas han sido estudiados (Watson et al. 1990), pero en México son poco conocidos y posiblemente uno de los menos conservados (MEA 2005, Zhou y Beck 2005). Los humedales en dolina dependen del agua subterránea, pueden alterarse al modificar el régimen hidrológico local o regional (Rohde et al. 2017), por ello su caracterización hidrogeomorfológica genera información para entender la dinámica, estructura y funcionamiento (Loheide y Booth 2011, Noe et al. 2013). Los humedales de la región hidrológica Yucatán norte son prioritarios, pero los humedales en dolina tienen nulo reconocimiento en el Inventario Nacional de Humedales (CONAGUA 2013). Por lo anterior, el objetivo fue caracterizar los humedales en dolina del norte Quintana Roo para conocer sus características hidrogeomorfológicas básicas.

\section{MATERIALES Y MÉTODOS}

\section{Área de estudio}

El área de estudio se localiza en la zona norte del estado de Quintana Roo, México, en los municipios de Benito Juárez y Puerto Morelos. El clima es cálido subhúmedo con lluvias en verano $(A w)$, temperatura media anual de $26^{\circ} \mathrm{C}$; precipitación anual de $1229 \mathrm{~mm}$ con lluvias abundantes de junio a octubre (INEGI 2016). El relieve es una llanura conformada por una secuencia de sedimentos calcáreos de origen marino del Terciario reciente, con secciones de reciente emergencia del agua donde afloran sedimentos del Cuaternario (INEGI 2016). Los suelos dominantes son rendzinas y litosoles (INEGI 2016). Se estudiaron cinco humedales en dolina: un humedal rural y cuatro urbanos de mayo a octubre de 2017 (Figura 1). El humedal en dolina de la zona rural se encuentra a $4.5 \mathrm{~km}$ al norte de Leona Vicario, municipio de Puerto Morelos conocido como El Corchal; mientras que los humedales urbanos se encuentran dentro de la ciudad de Cancún, municipio de Benito Juárez, conocidos como LC34, Hondonada, La Piedra y La Horquilla.

\section{Microtopografía}

La microtopografía de los humedales se midió por nivelación del relieve con un cuadro de aluminio de $1 \mathrm{~m}^{2}$ unido a un brazo móvil con un nivel de burbuja para determinar la diferencia en altura de un transepto, desde la parte elevada hasta la parte más baja del terreno. Se eligió un transepto radial del humedal, comenzando en el exterior y terminando en la parte de mayor inundación, debido a que los humedales en dolina presentan una circunferencia redonda o elíptica. La nivelación se realizó con respecto al terreno de mayor elevación y no con respecto al nivel del mar. En todos los casos, se registró la inundación promedio del humedal en centímetros entre los meses de mayo y octubre de 2017.

\section{Fisicoquímica del agua}

Los parámetros físicoquímicos de agua superficial e intersticial se midieron en cinco puntos de cada sitio de estudio. La temperatura $\left({ }^{\circ} \mathrm{C}\right), \mathrm{pH}$, conductividad eléctrica $\left(\mathrm{mS} \mathrm{cm} \mathrm{cm}^{-1}\right)$ y contenido de solidos disueltos totales $\left(\mathrm{g} \mathrm{L}^{-1}\right)$ se midió con una sonda Hanna ${ }^{\circledR}$ Temp/pH/EC/TDS (HI98129); mientras que el potencial de óxido reducción (mv) con una sonda Hanna ${ }^{\circledR}$ ORP/PH H198121. Las sondas se calibraron en campo con soluciones buffer y estándares de calibración. Se colectaron cinco muestras de agua para análisis de nutrientes en los mismos puntos donde se evaluaron los parámetros fisicoquímicos. El agua superficial se colectó de forma directa, y el agua intersticial a $15 \mathrm{~cm}$ de profundi- 


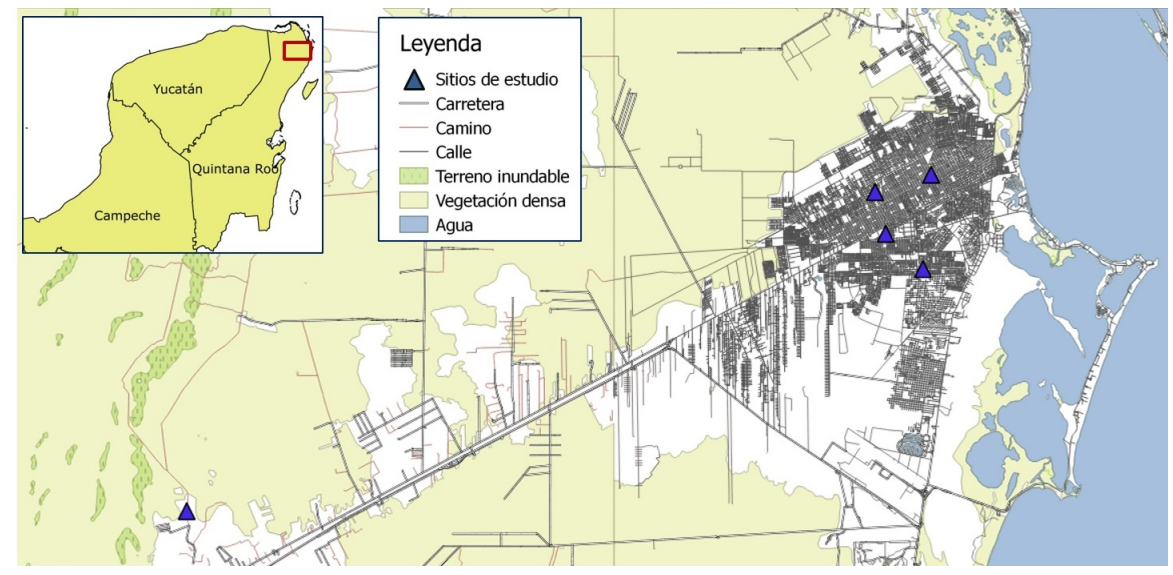

Figura 1. Localización de los humedales en dolina en estudio. Los humedales urbanos se localizan dentro de la ciudad de Cancún y el humedal de zona rural en la localidad de Leona Vicario.

dad del suelo con una manguera de tubo de cobre perforado de $1 / 2$ pulgada, conectada a una bomba peristáltica manual. Las muestras de agua se filtraron con un filtro de nitrocelulosa Pall ${ }^{\circledR}$ de 0.45 $\mu \mathrm{m}$ de tamaño de poro y se conservaron en frío (aproximadamente $4^{\circ} \mathrm{C}$ ) en botellas de polietileno de alta densidad Nalgene ${ }^{\circledR}$ de $60 \mathrm{ml}$. En el laboratorio de Fisicoquímicos de la Unidad de Ciencias del Agua del CICY se cuantificaron nitritos, amonio, ortofosfatos y nitratos con métodos espectrofotométricos en un espectrofotómetro UV-Vis Eppendorf BioSpectrometer ${ }^{\mathbb{R}}$. Los nitritos se cuantificaron con modificaciones del método de Strickland y Parsons (1972), usando tres mililitros de volumen de muestra y adaptando el rango de operación de 0.01 a $0.50 \mathrm{mg} \mathrm{N}-\mathrm{NO}_{2}^{-} \mathrm{L}^{-1}$. El ion amonio $\left(\mathrm{NH}_{4}^{+}\right)$se cuantificó con el método del salicilato (Bower y Holm-

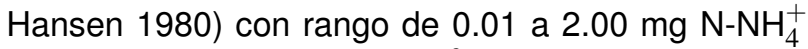
$\mathrm{L}^{-1}$. Los ortofosfatos $\left(\mathrm{PO}_{4}^{-3}\right)$ se cuantificaron con el método 365.3 de la USEPA (1978) con rango de 0.01 a $1.20 \mathrm{mg} \mathrm{P}-\mathrm{PO}_{4}^{-3} \mathrm{~L}^{-1}$. Se analizaron nitratos con la cuantificación rápida APHA 4500- $\mathrm{NO}_{3}^{-}$(APHA 2000). Las muestras que tuvieron valor de corrección por interferencia de materia orgánica superior al 10\% de la absorbancia no se reportan. Se analizaron los datos con una prueba ANOVA para detectar diferencias entre el origen de agua (superficial e intersticial) y humedales con el programa SPSS 13.0.

\section{Parámetros edáficos}

Los parámetros edáficos se midieron en los mismos sitios de toma de muestra de agua, con la metodología de Campos-Cascaredo y MorenoCasasola (2009). El cilindro de PVC usado para obtener núcleos de suelo fue de $5 \mathrm{~cm}$ de diámetro y $2 \mathrm{~cm}$ de altura $\left(39.3 \mathrm{~cm}^{3}\right)$. Después de secar a $60^{\circ} \mathrm{C}$ por $24 \mathrm{~h}$, se determinó el contenido gravimétrico del agua $\omega$ ( $\mathrm{g}$ agua $\mathrm{g}^{-1}$ suelo), densidad aparente $\mathrm{Da}(\mathrm{g}$ $\left.\mathrm{cm}^{-3}\right)$, contenido volumétrico de agua $\theta\left(\mathrm{cm}^{3} \mathrm{~cm}^{-3}\right)$, porosidad $\mathrm{P}$ y saturación $\mathrm{S}(\%)$ con las ecuaciones de la Tabla 1. Los datos se analizaron con el programa SPSS 13.0, aplicando la prueba ANOVA y la prueba post-hoc de Tukey (HSD, $\alpha=0.05$ ). En sub-muestras de suelo seco de cada humedal, se realizó un análisis cualitativo de elementos por espectroscopia de rayos $X$ (EDS) en un microscopio electrónico de barrido (MEB), para detectar la presencia de Mg, Al, Si, P, $\mathrm{S}, \mathrm{Cl}, \mathrm{K}, \mathrm{Ca}$ y $\mathrm{Fe}$. El soporte de las muestras fue una Double-Sided Carbon Tape (Electron Microscopy Sciences ${ }^{\circledR}$ ) por lo que el $\mathrm{C}$ y $\mathrm{O}$ se detectaron en la misma proporción en todas las muestras por lo que se eliminaron del análisis. Las condiciones del MEB para la adquisición de espectros fue de $35^{\circ}$ elevación de adquisición de espectro; 20.00 kv de aceleración, resolución mayor de 49 ev, con conteo variable de espectros. Los valores expresan el porcentaje de cada elemento respecto al total de la muestra. 
Tabla 1. Ecuaciones para medir los parámetros edáficos de suelo de humedales (Campos-Cascaredo y Moreno-Casasola 2009).

\begin{tabular}{lcc}
\hline Parámetro edáfico & Ecuación & Descripción \\
\hline Contenido gravimétrico del agua & $\omega=P_{h}-P_{s} / P_{s}$ & $\mathrm{P}_{h}=$ peso del suelo húmedo; $\mathrm{P}_{s}=$ peso del suelo seco. \\
Densidad aparente & $D_{a}=M / V$ & $\mathrm{M}=$ peso del suelo seco; $\mathrm{V}=$ volumen del cilindro $\left(39.3 \mathrm{~cm}^{3}\right)$. \\
Contenido volumétrico del agua & $\theta=\omega x D_{a} / D_{w}$ & $\omega=$ contenido gravimétrico del agua; $\mathrm{D}_{a}=$ densidad aparente; \\
Porosidad & $P=1-D_{a} / D_{r}$ & $\mathrm{D}_{a}=$ densidad aparente; $\mathrm{D}_{r}=$ densidad real del suelo $\left(\approx 2.54 \mathrm{~g} \mathrm{~cm}^{-3}\right)$. \\
Grado de saturación & $S=\theta / P$ & $\theta=$ contenido volumétrico de agua; $P=$ porosidad. \\
\hline
\end{tabular}

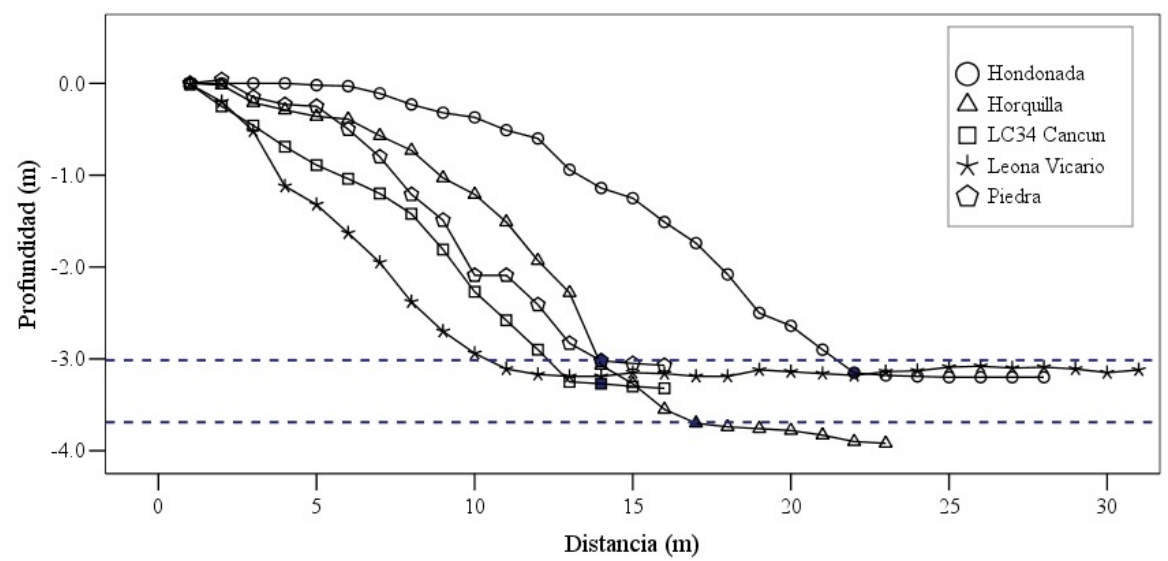

Figura 2. Perfil microtopográfico de los humedales en dolina del norte de Quintana Roo. Las líneas punteadas representan la mínima y la máxima profundidad registrada. Los puntos en color negro sólido indican el inicio de la zona inundada en cada sitio.

\section{Biomasa arbórea}

Se midió la circunferencia de entre 15 y 30 árboles con tronco mayor a $10 \mathrm{~cm}$ de circunferencia en cada humedal para calcular el volumen maderable con la fórmula de Smalian modificada, $\mathrm{V}=$ $\left(\mathrm{DAP}^{2}\right) x(\pi) x(0.4) x(\mathrm{~h})$, donde: DAP es el diámetro a la altura del pecho $(1.3 \mathrm{~m})$ y $\mathrm{h}$ la altura total del árbol. Obtenido el estimado de volumen maderable, se aplicó el método propuesto por la Alabama Forestry Comission para estimar la biomasa total aplicando un factor de conversión promedio de volumen maderable a biomasa de 1.19; después se obtuvo la biomasa en toneladas para luego estimar los kilogramos equivalentes de carbono por árbol con el factor de conversión de 0.5 de peso comparable de carbono en biomasa.

\section{RESULTADOS}

\section{Microtopografía}

Los humedales en dolina son depresiones de redondas a elípticas con roca expuesta y suelo poco o nada desarrollado, pendiente variable y en ocasiones paredes de roca vertical. Con entre 3.0 y 4.5 $\mathrm{m}$ de profundidad con respecto al terreno, inundación variable de 3.0 a $3.7 \mathrm{~m}$ de profundidad (Figura 2).

\section{Fisicoquímica del agua}

El análisis fisicoquímico del agua se muestra en la Tabla 2. Se observa que el agua intersticial tiene $\mathrm{pH}$ menor $(\mathrm{F}=8.08, \mathrm{p}=0.007)$ y conductividad eléctrica mayor que el agua superficial $(F=29.38, p<$ 0.001 ). Los sólidos disueltos totales fueron diferentes entre el agua superficial e intersticial $(F=27.62, p<$ 0.001). De acuerdo al potencial de óxido-reducción medido, las condiciones del agua son moderadamente reductoras, sin diferencia entre el agua superficial e intersticial. Los resultados del análisis de nitritos, amonio y ortofosfatos muestran que los nitritos estuvieron por debajo del límite de detección (menor a $0.01 \mathrm{mg} \mathrm{N}-\mathrm{NO}_{2}-\mathrm{L}^{-1}$ ); mientras que el amonio se detectó en todas las muestras $(F=7.71, p=0.01)$. La menor concentración de amonio se cuantificó en el humedal LC34, seguido de la Piedra y Leona Vicario, 
con las mayores concentraciones en la Hondonada y la Horquilla $(p \leq 0.05)$. Los ortofosfatos se midieron en concentraciones mayores a $0.2 \mathrm{mg} \mathrm{P}-\mathrm{PO}_{4}^{-3} \mathrm{~L}^{-1} \mathrm{y}$ no se encontraron diferencias entre los valores cuantificados entre agua superficial e intersticial. No se reportan los nitratos debido a que la corrección por interferencia de materia orgánica fue mayor al $10 \%$ de la lectura, por lo que no se consideran confiables.

\section{Parámetros edáficos}

El suelo de los humedales en dolina siempre estuvo saturado, los parámetros edáficos sugieren que los suelos son hidromorfos con alta capacidad de retención de agua y porosidad, con saturación mayor al $70 \%$. Con excepción del contenido gravimétrico, el resto de los parámetros fueron diferentes entre sitios (Tabla 3). El análisis por espectroscopia de rayos $X$ (EDS) de la fracción mineral del suelo mostró que el elemento más abundante en los humedales urbanos fue el calcio (53.8 a $87.5 \%$ ); mientras que en el humedal de la zona rural los mayores componentes fueron sílice (36.4\%) y aluminio (24.4\%). El fósforo y azufre solo se detectaron en un humedal urbano (LC $34)$ y el hierro fue mayor en el humedal rural $(19.8 \%$, Tabla 4). Las fotografías de microscopio electrónico de barrido muestran la textura de grano y la distribución de partículas en el suelo de los humedales (Figura 3).

\section{Biomasa arbórea}

Se estimó la biomasa arbórea como el equivalente en kilogramos de carbono por árbol a partir del volumen maderable de los árboles medidos en cada humedal. La biomasa arbórea es diferente entre sitio, con entre 0.01 y $0.30 \mathrm{~kg}$ de carbono por árbol (Figura 4). Se observaron valores atípicos en los humedales urbanos LC34 $(6.90 \mathrm{~kg} \mathrm{C})$ y La Horquilla (1.9 $\mathrm{kg} \mathrm{C})$ que corresponden con árboles de gran fuste del género Ficus. Se observó la presencia de especies del genero Ficus, zapote (Manilkara zapota), palma chit (Thrinax radiata), palma guano ( $\mathrm{Sa}$ bal mexicana), almendro (Terminalia catapa) y Anona glabra en los humedales de Benito Juárez; mientras que el humedal El Corchal es monotípico dominado por Anona glabra.

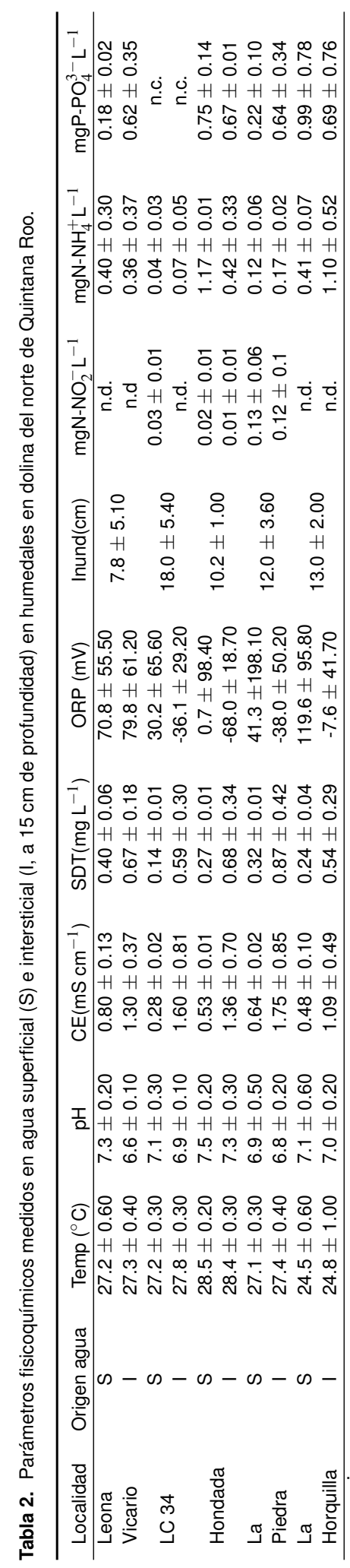


Cejudo y Herrera-Caamal

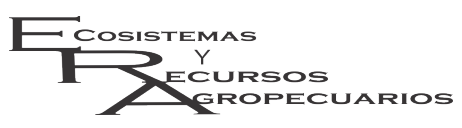

Humedales en dolina: ecosistemas poco conocidos

Ecosist. Recur. Agropec. 6(17):207-218,2019

Tabla 3. Parámetros edáficos medidos en suelo de humedales en dolina del norte de Quintana Roo.

\begin{tabular}{cccccc}
\hline Localidad & $\omega\left(\mathrm{gr} \mathrm{gr}^{-1}\right)$ & $\mathrm{Da}\left(\mathrm{g} \mathrm{cm}^{-3}\right)$ & $\theta\left(\mathrm{cm}^{3} \mathrm{~cm}^{-3}\right)$ & $\mathrm{P}(\%)$ & $\mathrm{S}(\%)$ \\
\hline Leona Vicario & $2.3 \pm 0.09$ & $0.31 \pm 0.13^{b}$ & $0.81 \pm 0.15^{a}$ & $88 \pm 50^{a}$ & $1.15 \pm 0.04^{a b}$ \\
LC 34 & $1.96 \pm 0.95$ & $0.41 \pm 0.24^{b}$ & $0.60 \pm 0.07^{b}$ & $84 \pm 90^{a}$ & $0.73 \pm 0.15^{b}$ \\
Hondonada & $1.35 \pm 0.63$ & $0.77 \pm 0.30^{a b}$ & $0.86 \pm 0.18^{a b}$ & $69 \pm 12^{a}$ & $1.3 \pm 0.42^{a b}$ \\
La Piedra & $1.94 \pm 1.00$ & $0.44 \pm 0.17^{b}$ & $0.69 \pm 0.12^{a b}$ & $82 \pm 80^{a}$ & $0.84 \pm 0.08^{a b}$ \\
La Horquilla & $0.45 \pm 0.20$ & $1.36 \pm 0.35^{a}$ & $0.55 \pm 0.10^{b}$ & $34 \pm 11^{b}$ & $1.7 \pm 0.58^{a}$ \\
\hline
\end{tabular}

Promedio \pm desviación estándar $(n=5)$. Las letras indican diferencias entre muestras (Tukey HSD $\alpha=0.05)$.

Tabla 4. Análisis elemental por espectroscopia de rayos $X$ (EDS) de la fracción mineral del suelo de humedales en dolina del norte de Quintana Roo.

\begin{tabular}{lccccc}
\hline & \multicolumn{5}{c}{ Humedal } \\
\cline { 2 - 6 } Elemento & Leona Vicario & LC 34 & La Hondonada & La Horquilla & La Piedra \\
\hline Mg (\%) & 1.40 & - & 2.40 & 1.50 & 5.60 \\
$\mathrm{Al}(\%)$ & 24.40 & 14.90 & 14.90 & 4.60 & 8.00 \\
$\mathrm{Si}(\%)$ & 36.40 & 16.60 & 23.50 & 5.40 & 12.80 \\
$\mathrm{P}(\%)$ & - & 3.50 & - & - & - \\
$\mathrm{S}(\%)$ & - & 2.00 & - & - & - \\
$\mathrm{Cl}(\%)$ & - & - & - & - & 4.00 \\
$\mathrm{~K}(\%)$ & 0.90 & - & 1.10 & - & - \\
$\mathrm{Ca}(\%)$ & 17.20 & 63.00 & 53.80 & 87.50 & 67.00 \\
$\mathrm{Fe}(\%)$ & 19.80 & - & 4.20 & 1.00 & 2.60 \\
\hline
\end{tabular}

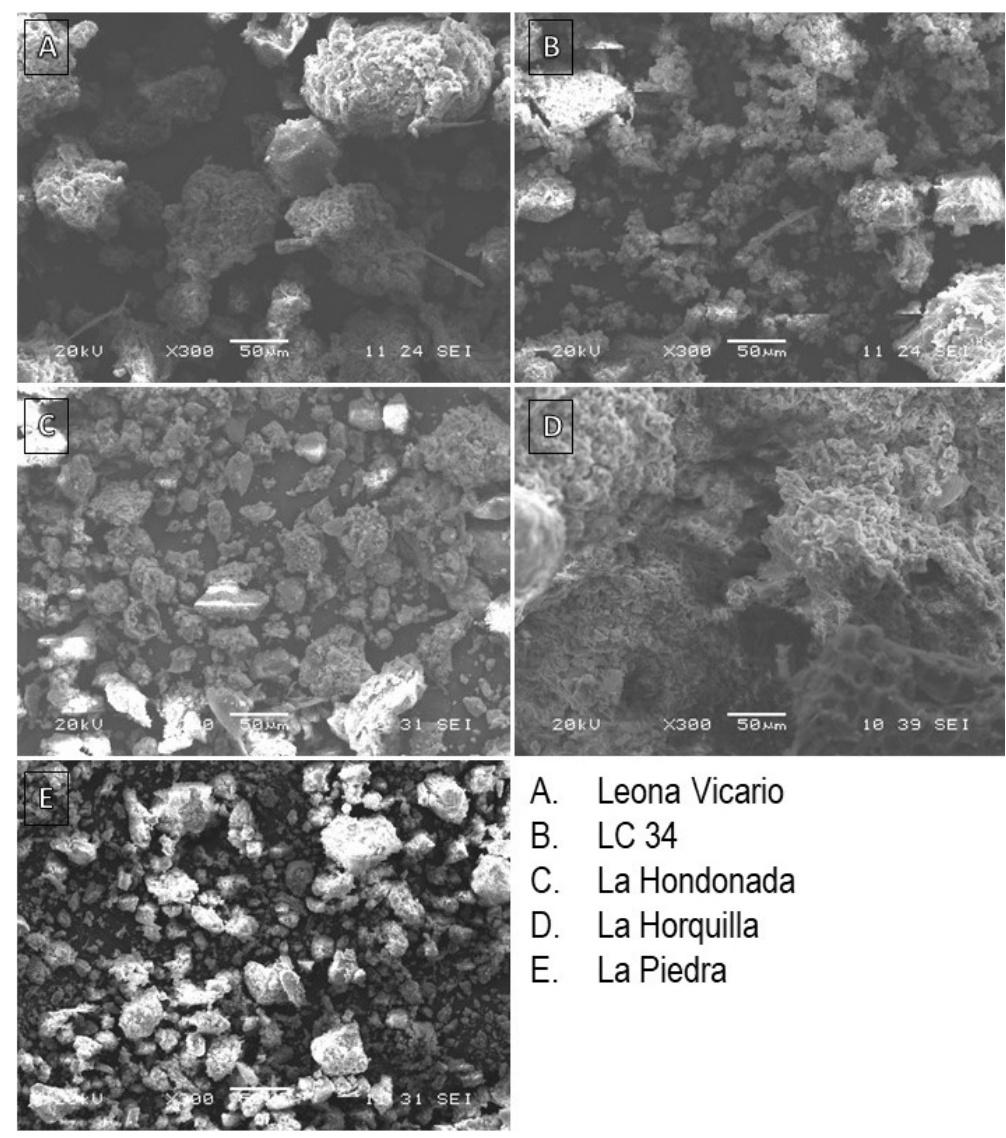

Figura 3. Fotografías de microscopio electrónico de barrido (MEB) de muestras de suelos de los humedales. 


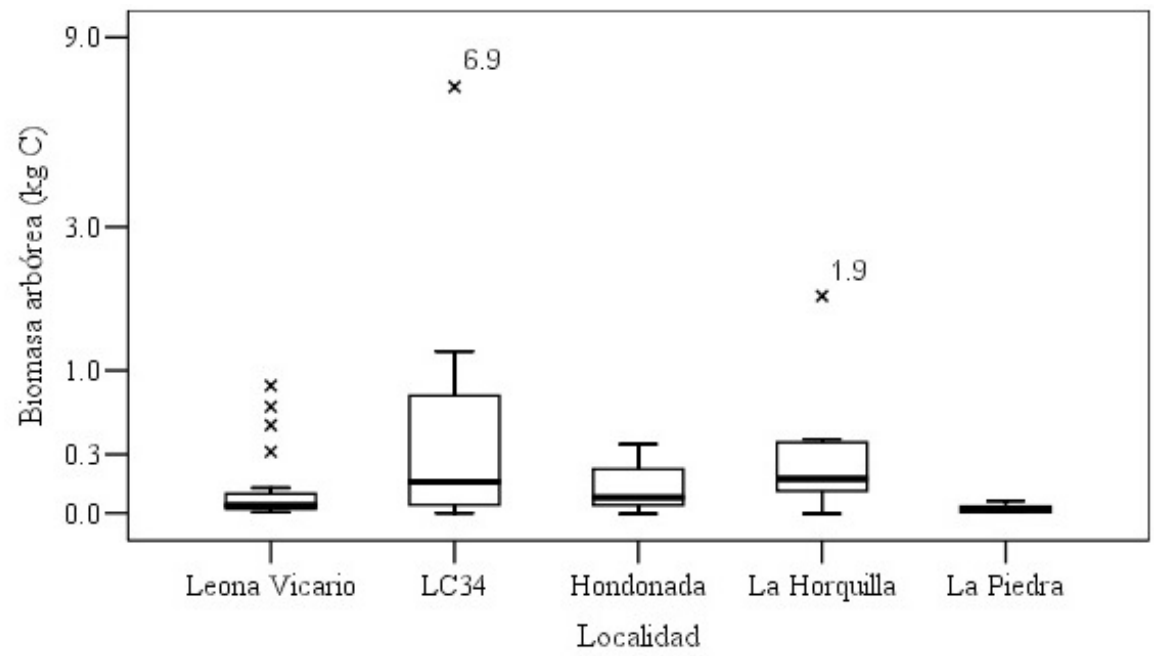

Figura 4. Biomasa arbórea $(\mathrm{kg} \mathrm{C})$ por árbol estimada en humedales en dolina del norte de Quintana Roo.

\section{Clasificación de los humedales en dolina}

De acuerdo con la clasificación de humedales de la CONAGUA, los humedales en dolina del norte de Quintana Roo son sistemas palustres en depresión con régimen de estacional a intermitentemente inundado y saturación permanente, siendo unidades primarias tipo $\mathrm{P} / \mathrm{b} / \mathrm{h}$. Al utilizar los descriptores de escala 1:20 000, los humedales son depresiones húmedas en clima cálido tipo leptoescala ( 1100 a $2800 \mathrm{~m}^{2}$ de superficie), de forma ovoide o redonda, con organización interna de vegetación de zoniforme a gradiforme, dominadas por formaciones arbóreas y presencia de herbáceas emergentes. El sustrato de los humedales es fondo rocoso con secciones de fondo no consolidado. En los humedales LC34, La Piedra, La Horquilla y Hondonada el uso de suelo predominante de los alrededores es urbano. Mientras que el humedal de Leona Vicario es propiedad ejidal con uso forestal y pequeñas parcelas agrícolas.

\section{DISCUSIÓN}

La fisiografía de la península de Yucatán tiene variación microtopográfica que permite el establecimiento de humedales en dolinas (BautistaZuñiga et al. 2002). Con los perfiles microtopográficos se verificó la forma ovalada a redonda de la doli- na, profundidad entre 3 y $4 \mathrm{~m}$ con respecto al terreno, el declive de las paredes puede ser con pendiente suave o abrupta. Debido a que los muestreos se realizaron en la época de secas (mayo) y en lluvias (octubre) se observó que la temporalidad y profundidad de inundación es variable con suelo siempre saturado; lo que define la cobertura vegetal dominante, las condiciones del suelo y el tipo de humedal (Moreno-Casasola et al. 2012, CONAGUA 2013). Los humedales en dolina ocupan superficies menores de $0.5 \mathrm{ha}$, rodeados de tierras elevadas, lo que los convierte en humedales aislados (Tiner 2003), pero conectados con otros por aguas subterráneas, por lo que forman parte del sistema hidrológico regional, importantes para la recarga de agua subterránea y la regulación de inundaciones (Winter y LeBaugh 2003).

$\mathrm{El} \mathrm{pH}$ ligeramente ácido, los sólidos disueltos totales y la conductividad eléctrica mayor en el agua intersticial comparada con el agua superficial, sugieren que el agua de poro contiene mayor cantidad de sólidos disueltos y ácidos orgánicos. La cantidad de sólidos disueltos totales es similar a lo reportado en agua de pozos de extracción de la ciudad de Cancún (CONAGUA 2018), lo que indica que el agua subterránea es la fuente de agua que abastece a los humedales en dolina. La temperatura del agua superficial varió con respecto a la densidad de la 
cobertura vegetal y penetración de luz solar. A pesar de la poca oscilación en la temperatura del agua, es posible que estas variaciones tengan influencia en la tasa y velocidad de las reacciones químicas que ocurren en el suelo, e influyan en la actividad biológica y las condiciones de oxidación-reducción del suelo y agua (Seybold et al. 2002). El potencial de óxido-reducción del agua superficial e intersticial fue de moderadamente reductor a reductor, por lo que se espera que domine el metabolismo microbiano facultativo (Falkowski et al. 2008). El potencial de óxido-reducción menor a cero y cercano a $100 \mathrm{mv}$ ha sido descrito en humedales riparios (Seybold et al. 2002) y humedales arbóreos del Golfo de México (Moreno-Casasola et al. 2012). En éstas condiciones de óxido-reducción, Drake et al. (2009) mencionan que varios procesos anaerobios pueden ocurrir de manera simultánea debido a la alta disponibilidad de materia orgánica, por lo que se espera que los humedales en dolina tengan elevadas concentraciones de amonio que no es completamente oxidado. Las condiciones reductoras del agua ayudan a explicar la detección del ion amonio de todas las muestras, con mayor concentración en el agua intersticial, lo que se puede deber a la continua mineralización de materia orgánica y menor oxidación del amonio por la baja tensión de oxígeno. Los nitritos estuvieron por debajo del límite de detección $(0.01 \mathrm{mg}$ $\mathrm{N}-\mathrm{NO}_{2}^{-} \mathrm{L}^{-1}$ ), posiblemente debido a lenta oxidación del amonio, o reducción a otros compuestos nitrogenados (Van-Cleemput y Samater 1996), ser aceptor de electrones (Ettwig et al. 2010) o posible oxidación anaerobia de amonio (Zhu et al. 2010). Debido a que no se pudieron medir los nitratos, no se sabe si se están acumulando o hay desnitrificación. Al respecto, Marton et al. (2015) proponen entender la biogeoquímica de los humedales en dolina con las especies disueltas de nitrógeno, ya que son reactores biogeoquímicos del paisaje con importancia para la calidad del agua.

El fósforo comúnmente se encuentra en complejos minerales y se precipita en condiciones de abundante calcita (Shenker et al. 2005, Mitsch y Gosselink 2007), por lo que se encuentra en mayor concentración en la matriz mineral y no se espera la concentración de ortofosfatos observada en el agua (mayor a $0.2 \mathrm{mg} \mathrm{P} \mathrm{PO}_{4}^{-3} \mathrm{~L}^{-1}$ ). El fósforo puede ser disuelto en condiciones reductoras (Shenker et al. 2005) y quedar libre en solución, lo que puede estar ocurriendo en los humedales en dolina. Debido a que los resultados provienen de un muestreo por estación (secas y lluvias), es necesario monitorear las condiciones de oxidación-reducción del suelo y agua para comprender la biogeoquímica de éstos ecosistemas.

De acuerdo con el contenido gravimétrico y volumétrico, y el porcentaje de saturación del suelo usados como indicadores de contenido de humedad, todos los humedales tienen alta capacidad de retención de agua. Los humedales urbanos tuvieron mayor densidad aparente comparados con los humedales de zona rural, posiblemente por la compactación por las actividades humanas. El humedal urbano La Horquilla tuvo los valores más bajos de contenido de agua y porosidad, además de mayor densidad aparente. Éste sitio es un parque municipal donde se realizan actividades recreativas. El sitio tiene dos estanques que funcionan como almacén de drenaje pluvial; por lo que en temporada de lluvias, el nivel freático se eleva y el agua subterránea se mezcla con el agua pluvial colectada en los estanques, siendo una potencial entrada de nutrientes al humedal. De acuerdo con Bautista et al. (2011) las geoformas de tipo fluvio-palustre comúnmente tienen suelos tipos Gleysol, Histosol, Leptosol y Solonchack; los dos primeros en humedales por los procesos de ausencia de oxígeno y alto contenido de materia orgánica. El análisis de la fracción mineral del suelo muestra que el calcio es el mineral más abundante en los humedales en dolina, lo que se relaciona con el origen de la matriz rocosa, dominada por calcitas, dolomías y coquina (Perry et al. 2002). El magnesio es otro elemento común en roca sedimentaria de la península de Yucatán (Perry et al. 2002), pero se encontró solo en cuatro humedales.

Los humedales urbanos tuvieron mayor contenido de calcio y el rural la mayor fracción de sílice. Lo que no se esperaba, debido a que la roca dominante en la región es caliza. Pero es posible que la alta proporción de sílice se deba a intemperización de roca. Estudios previos reportan que el sílice es 


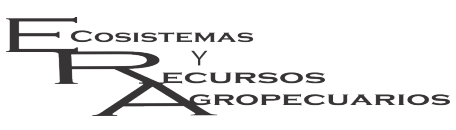

Cejudo y Herrera-Caamal

Humedales en dolina: ecosistemas poco conocidos

Ecosist. Recur. Agropec. 6(17):207-218,2019

un indicador de origen terrestre de agua subterránea (Hernández-Terrones et al. 2011). Otro mineral es el aluminio, elemento importante que en suelos con condiciones de acidez pueden generar la especie tóxica $\mathrm{Al}^{+3}$, de alta movilidad en el ambiente (MuñozSánchez et al. 2015). El contenido de hierro, potasio, fósforo y cloro fue variable por sitio, lo que puede indicar la mineralogía e interacción agua-roca (Perry et al. 2002).

Los humedales urbanos estuvieron dominados por especies del género Ficus, Manilkara zapota, Terminalia catapa y la palma Thrinax radiata. El humedal rural Leona Vicario es monotípico dominado por Anona glabra, la biomasa arbórea comúnmente fue menor de $0.30 \mathrm{~kg}$ de carbono por árbol, con mayor variabilidad en el humedal urbano LC34. Estudios en humedales tropicales cársticos reportan que la mayor reserva de carbono no necesariamente se encuentra en la biomasa, sino en el suelo (Adame et al. 2013). Es importante mencionar que la mayor parte de las mediciones se completaron en los arboles establecidos en la porción de menor inundación, menos de 30 $\mathrm{cm}$ de profundidad, por lo que no se conoce el volumen maderable de los árboles que están en la zona profunda o en las zonas de sedimento menos consolidado y de difícil acceso. Los humedales en dolina son unidades de paisaje distinguibles por la saturación de suelo y la vegetación dominante. Proveen los servicios ecosistémicos de amortiguamiento de inundaciones, recarga del acuífero, y mejoran la calidad de agua y de recreación. Al ser ecosistemas geográficamente aislados, pero hidrológicamente conectados dentro de una zona urbana, pueden ser sitios importantes de movilización de solutos y dispersión de contaminantes.

\section{CONCLUSIONES}

Los humedales son geoformas ovoides o redondas de entre 3 y $4 \mathrm{~m}$ de profundidad, superficie menor a 0.50 ha dominada por vegetación arbórea, estacional a intermitentemente inundado y de sustrato rocoso con secciones de sedimento no consolidado del tipo $\mathrm{P} / \mathrm{b} / \mathrm{h}$. El agua tuvo condiciones reductoras, con presencia de amonio y ortofosfatos en concentración mayor a $0.2 \mathrm{mg} \mathrm{N} \mathrm{L} \mathrm{m}^{-1}$ y $0.2 \mathrm{mg}$ $\mathrm{P} \mathrm{L}^{-1}$; con suelos hidromorfos de alta capacidad de retención de agua, porosidad y saturación mayor a $70 \%$, elevado contenido de calcio, sílice y aluminio. La biomasa arbórea oscila entre 0.01 y $0.30 \mathrm{~kg}$ de carbono por árbol.

\section{AGRADECIMIENTOS}

Al Ayuntamiento de Benito Juárez, Quintana Roo por el permiso (DGE/DPA/2748/2017); a la Comisaría Ejidal de Leona Vicario, Municipio de Puerto Morelos por el acceso al sitio El Corchal. A la IQ Silvia B. Andrade Canto por los análisis de espectroscopia de rayos $X$ e imágenes del Microscopio Electrónico de Barrido del CICY. A la Quim. Daniela Ortega e Ing. Amb. Irainit Valencia por su apoyo en laboratorio.

\section{LITERATURA CITADA}

Adame MF, Kauffman JB, Medina I, Gamboa JN, Torres O, Caamal J et al. (2013) Carbon stocks of tropical coastal wetlands within the karstic landscape of the Mexican Caribbean. PLoS ONE 8:e56569.

Aguilar Y, Bautista F, Mendoza ME, Frausto O, Ihl T (2016) Density of karst depressions in Yucatán state, Mexico. Journal of Cave and Karst Studies 78: 51-60.

AFC (2018) Carbon sequestration. Alabama Forestry Commission. http://www.forestry.state.al.us/HowMuchCarbonHaveYourTreesStored.aspx?bv=5\&s=0). Fecha de consulta 2 de febrero 2018.

APHA (2017) Method 4500-NO - : Nitrogen (Nitrate). Standard Methods for the Examination of Water and Wastewater. Doi: 10.2105/SMWW.2882.089 
Bautista F, Palacio-Aponte G, Quintana P, Zinck JA (2011) Spatial distribution and development of soils in tropical karst areas from the Peninsula of Yucatan, Mexico. Geomorphology 35: 308-321.

Bautista-Zúñiga F, Jiménez-Osornio J, Navarro-Alberto J, Manu A, Lozano R (2002) Micro-Relief and soil color as diagnostic properties in carstic leptosols. Terra Latinoamericana 21: 1-11.

Beltram G (2016) Karst Wetlands. In: Finlayson C, Milton G, Prentice R, Davidson N (eds). The wetland book. Springer Netherlands. pp: 1-17.

Bower CE, Holm-Hansen T (1980) A salicylate-hypochlorite method for determining ammonia in seawater. Canadian Journal of Fisheries and Aquatic Sciences 37: 794-798.

Campos-Cascaredo A, Moreno-Casasola P (2009) Suelos hidromorfos. In: Moreno-Casasola P, Warner B (eds) Breviario para describir, observar y manejar humedales. Serie Costa Sustentable no 1. RAMSAR, Instituto de Ecología. CONANP, US Fish and Wildlife Service, US State Department. Xalapa, Ver. México. pp: 111-130.

CONAGUA (2013) Lineamientos para la clasificación de humedales. Comisión Nacional del Agua, Subdirección General Técnica, Gerencia de Calidad del Agua. https://www.gob.mx/cms/uploads/attachment/file/165385/ Clasificaci_n.pdf. Fecha de consulta: 30 enero 2018.

CONAGUA (2018) Sistema Nacional de Información del Agua. Comisión Nacional del Agua, Gerencia de Coordinación Interinstitucional. http://sina.conagua.gob.mx/sina/tema.php?tema=calidadAgua\&ver=reporte\&o=4\& $\mathrm{n}=$ nacional. Fecha de consulta: 7 diciembre 2018.

Costanza R, de Groot R, Sutton P, Van der Ploeg S, Anderson SJ, Kubiszewski I, et al. (2014) Changes in the global value of ecosystem services. Global Environmental Change 26: 152-158.

Cronk JK, Mitsch WJ (1994) Periphyton productivity on artificial and natural surfaces in constructed freshwater wetlands under different hydrologic regimes. Aquatic Botany 48: 325-341.

Drake HL, Horn MA, Wüst PK (2009) Intermediary ecosystem metabolism as a main driver of methanogenesis in acidic wetland soil. Environmental Microbiology Reports 1: 307-318.

Ettwig KF, Butler MK, Le-Paslier D, Pelletier E, Mangenot S, Kuypers MM, et al. (2010) Nitrite-driven anaerobic methane oxidation by oxygenic bacteria. Nature. 464: 543-548.

Falkowski PG, Fenchel T, Delong EF (2008) The microbial engines that drive Earth's biogeochemical cycles. Science 320: 1034-1039.

Gutiérrez F, Galve JP, Guerrero J, Lucha P, Cendrero A, Remondo J, et al. (2007) The origin, typology, spatial distribution and detrimental effects of the sinkholes developed in the alluvial evaporite karst of the Ebro River valley downstream of Zaragoza city (NE Spain). Earth Surface Processes and Landforms 32: 912-928.

Hernández-Terrones L, Rebolledo-Vieyra M, Merino-Ibarra M, Soto M, Le-Cossec A, Monroy-Ríos E (2011) Groundwater pollution in a karstic region (NE Yucatan): baseline nutrient content and flux to coastal ecosystems. Water, Air, \& Soil Pollution 218: 517-528.

INEGI (2016) Anuario estadístico y geográfico de Quintana Roo 2016. Instituto Nacional de Estadística y Geografía. México. 407p.

Kresic N (2013) Water in karst. McGraw-Hill. Estados Unidos de América. 707p.

Loheide SP, Booth EG (2011) Effects of changing channel morphology on vegetation, groundwater, and soil moisture regimes in groundwater-dependent ecosystems. Geomorphology 126: 364-376. 
Marton JM, Creed IF, Lewis DB, Lane CR, Basu NB, Cohen M, et al. (2015) Geographically isolated wetlands are important biogeochemical reactors on the landscape. Bioscience 65: 408-418.

MEA (2005) Ecosystems and human well-being: Wetlands and water - synthesis. Millennium Ecosystem Assessment. World Resources Institute, Washington, DC. Estados Unidos de América. 68p.

Mitsch WJ, Gosselink JG (2000) The value of wetlands: importance of scale and landscape setting. Ecological Economics 35: 25-33.

Mitsch WJ, Gosselink JG (2007) Wetlands. $4^{\circ}$ Ed. John Wiley \& Sons. Estados Unidos de América. 547p.

Mitsch WJ, Bernal B, Hernández ME (2015) Ecosystem services of wetlands. International Journal of Biodiversity Science, Ecosystem Services \& Management 11: 1-4.

Moreno-Casasola P, Infante Mata D, López Rosas H (2012) Tropical freshwater swamps and marshes. In: Batzer D, Baldwin A (eds). Wetlands habitats of North America: ecology and conservation. University of California Press. Estados Unidos de América. pp: 267-282.

Muñoz-Sánchez A, Domínguez-Domínguez R, Hernández-Sotomayor T (2015) Characterizing the relationship between aluminum content and $\mathrm{pH}$ in coffee (Coffea arabica L.) crop soils in Mexico. International Journal of Agriculture and Crop Science 8: 39-46.

Noe GB, Hupp CR, Rybicki NB (2013) Hydrogeomorphology influences soil nitrogen and phosphorus mineralization in floodplain wetlands. Ecosystems 16: 75-94.

Palacio Aponte AG, Noriega Trejo R, Zamora Crescencio P (2002) Caracterización físico-geográfica del paisaje conocido como "bajos inundables": El caso del Área Natural Protegida Balamkín, Campeche. Investigaciones geográficas 49: 57-73.

Perry E, Velazquez-Oliman G, Marin L (2002) The hydrogeochemistry of the karst aquifer system of the northern Yucatan Peninsula, Mexico. International Geology Review 44: 191-221.

Ramsar (2015) Nota informativa Ramsar 7 - Estado de los humedales del mundo y de los servicios que prestan a las personas: una recopilación de análisis recientes. www.ramsar.org/library. Fecha de consulta: 2 de febrero de 2018.

Ramsar (2018) Ramsar country profiles México. https://www.ramsar.org/wetland/mexico. Fecha de consulta: 2 de febrero de 2018.

Rohde MM, Froend R, Howard J (2017) A global synthesis of managing groundwater dependent ecosystems under sustainable groundwater policy. Groundwater 55: 293-301.

Serran JN, Creed IF (2016) New mapping techniques to estimate the preferential loss of small wetlands on prairie landscapes. Hydrological Processes 30: 396-409.

Seybold CA, Mersie W, Huang J, McNamee C (2002) Soil redox, pH, temperature, and water-table patterns of a freshwater tidal wetland. Wetlands 22: 149-158.

Shenker M, Seitelbach S, Brand S, Haim A, Litaor MI (2005) Redox reactions and phosphorus release in reflooded soils of an altered wetland. European Journal of Soil Science 56: 515-525.

Strickland JD, Parsons TR (1972). Determination of reactive nitrite. Fisheries Research Board of Canada Bulletin: A Practical Handbook of Seawater Analysis 167: 71-75.

Tiner RW (2003) Geographically isolated wetlands of the United States. Wetlands 23: 494-516. 
Torrescano-Valle N, Folan WJ (2015) Physical settings, environmental history with an outlok on global change. In: Islebe GA, Calmé S, León Cortés JL, Schmook B (eds). Biodiversity and conservation of the Yucatan Peninsula. Springer International Publishing. Switzerland. pp: 9-37.

USEPA (1978) Method 365.3: Phosphorous, All Forms (Colorimetric, Ascorbic Acid, Two Reagent). United States Environmental Protection Agency. https://www.epa.gov/sites/production/files/2015-08/documents/method_ 365-3_1978.pdf. Fecha de consulta: 26 de noviembre 2017.

Van-Cleemput O, Samater AH (1996) Nitrite in soils: Accumulation and role in the formation of gaseous $\mathrm{N}$ compounds. Fertilizer research. 45: 81-89.

Watson J, Stedje D, Barcelo M, Stewart M (1990) Hydrogeologic investigation of cypress dome wetlands in well field areas north of Tampa, Florida. In: Proc. Focus Eastern Conference, National Water Well Assoc. Dublin, OH, USA. pp: 163-176.

Winter TC, LaBaugh JW (2003) Hydrologic considerations in defining isolated wetlands. Wetlands 23: 532-540.

Zhou W, Beck BF (2005) Roadway construction in karst areas: management of stormwater runoff and sinkhole risk assessment. Environmental Geology 47: 1138-1149.

Zhu G, Jetten MSM, Kuschk P, Ettwig KK, Yin C (2010) Potential roles of anaerobic ammonium and methane oxidation in the nitrogen cycle of wetland ecosystems. Applied Microbiology and Biotechnology. 86: 10431055. 